Case Report

\title{
Hypofractionated Stereotactic Radiotherapy after Transarterial Chemoembolisation Failure in an Unresectable Hepatocellular Carcinoma: A Case Presentation
}

\author{
Francesco Fiorica, ${ }^{1}$ Carlo Greco, ${ }^{2}$ Sergio Boccia, ${ }^{3}$ Sergio Sartori, ${ }^{4}$ Antonio Stefanelli, \\ Francesco Cartei, ${ }^{1}$ and Stefano Ursino ${ }^{1,2}$ \\ ${ }^{1}$ Department of Radiation Oncology, University Hospital "S. Anna," Corso Giovecca 203, 44100 Ferrara, Italy \\ ${ }^{2}$ Department of Radiation Oncology, University Hospital "S. Chiara," Via Roma 67, 56126 Pisa, Italy \\ ${ }^{3}$ Department of Gastroenterology, University Hospital “S. Anna," Via Aldo Moro 8, 44124 Ferrara, Italy \\ ${ }^{4}$ Department of Internal Medicine, Section of Interventional Ultrasound, University Hospital "S. Anna," \\ Via Aldo Moro 8, 44124 Ferrara, Italy \\ Correspondence should be addressed to Stefano Ursino; stefano.ursino@med.unipi.it
}

Received 28 January 2013; Accepted 14 February 2013

Academic Editors: E. Hadziyannis and N. Terayama

Copyright (C) 2013 Francesco Fiorica et al. This is an open access article distributed under the Creative Commons Attribution License, which permits unrestricted use, distribution, and reproduction in any medium, provided the original work is properly cited.

\begin{abstract}
Introduction. Transarterial chemoembolization is the first-line treatment in unresectable hepatocellular carcinoma. There is no standard treatment after transarterial chemoembolization failure. We report the case of a patient with advanced hepatocellular carcinoma who showed a complete response and a long cancer control with hypofractionated stereotactic radiotherapy after transarterial chemoembolization failure. Case Presentation. A 70-year-old Caucasian woman was treated with transarterial chemoembolization for advanced hepatocellular, but no cancer control was obtained. A hypofractionated stereotactic radiotherapy was planned delivering $40 \mathrm{~Gy}$ in 5 fractions. A dramatic reduction in alpha-fetoprotein was observed. Contrast-enhanced ultrasonography at 1 and 2 months showed large necrotic areas. Computerised tomography scan showed a 90\% objective tumour response, then a complete remission at 3 and 6 months after treatment, respectively. Status of patient remained unchanged for 2 years. Conclusions. Hypofractionated stereotactic radiotherapy can improve survival and prognosis of unresectable hepatocellular carcinoma patient.
\end{abstract}

\section{Introduction}

Hepatocellular carcinoma (HCC) is a very heterogeneous disease, and the management of therapeutic approach may be variable and strongly related to patient's liver dysfunction as well as tumour stage [1]. A patient affected by intermediate or advanced stage HCC is only candidate for a palliative treatment. Transarterial chemoembolization (TACE) is standard treatment of unresectable HCC [2]; however, prognosis of these patients remains dismal, especially when TACE fails. Hypofractionated stereotactic radiotherapy (HSRT) can lead to a significant benefit for patients affected by intermediate or advanced HCC, alone or combined with TACE [3].
We report the case of a patient with advanced HCC who showed a complete response and a long cancer control with HSRT after TACE failure.

\section{Case Presentation}

A 70-year-old woman, with a history of alcoholic cirrhosis was admitted to our hospital because a liver mass had been discovered by ultrasonography scan. Her Eastern Cooperative Oncology Group (ECOG) performance score was 1. Physical examination showed no palpable mass, abdominal distension, or weight loss. Laboratory details showed white blood cell $5,980 / \mu \mathrm{L}$; red blood cell 


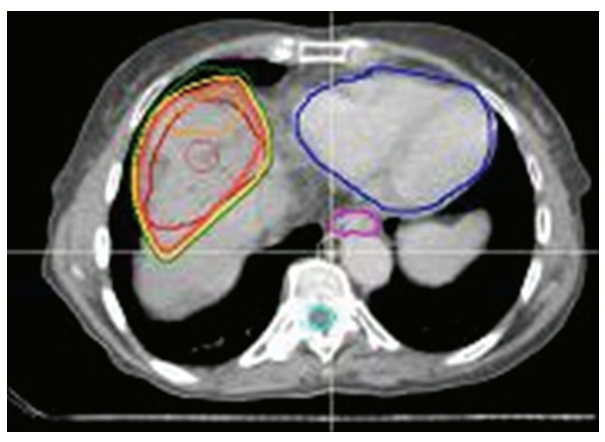

(a)

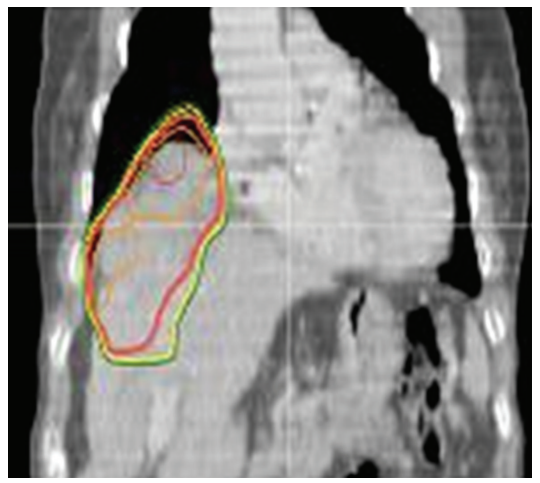

(c)

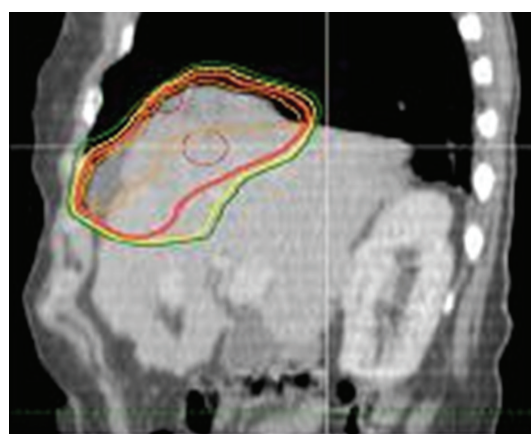

(b)

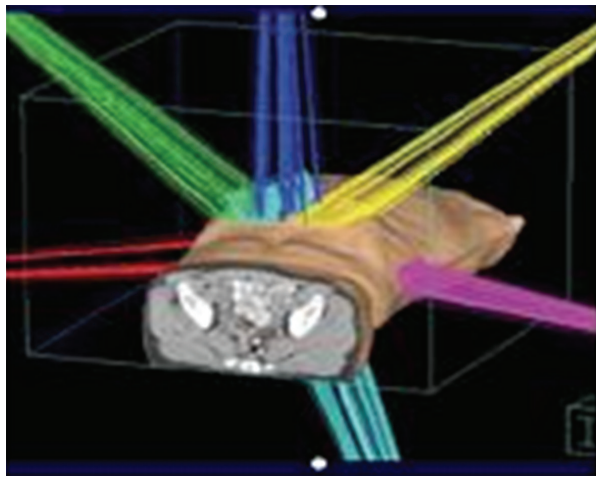

(d)

FIGURE 1: HSRT treatment plan: the axial (a), sagittal (b), coronal (c), and three-dimensional view (d). Red line: 100\% isodose line; Yellow line: $95 \%$ isodose line; green line: $80 \%$ isodose line.

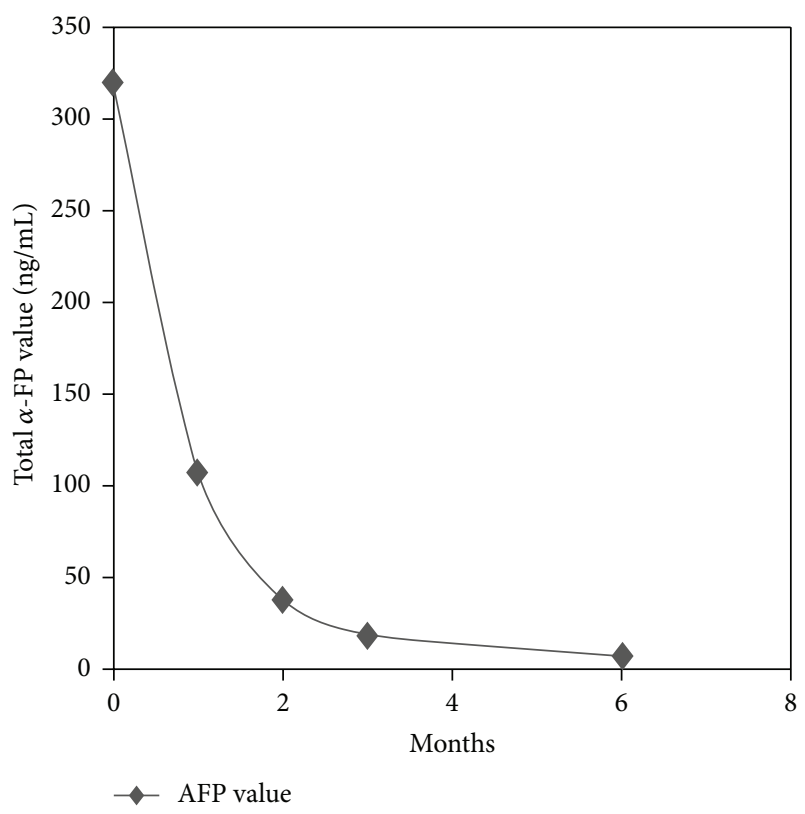

FIGURE 2: Changes in $\alpha$-FP levels after HSRT.

$4,24 \times 10^{6} / \mu \mathrm{L}$; haemoglobin $10,8 \mathrm{~g} / \mathrm{dL}$; hematocrit $35 \%$; platelet $137,000 / \mu \mathrm{L} ;$ INR 1,11 ; urea nitrogen $26 \mathrm{mg} / \mathrm{dL}$; creatinine $0,73 \mathrm{mg} / \mathrm{dL}$; total protein $7,8 \mathrm{~g} / \mathrm{dL}$; albumin $3,5 \mathrm{~g} / \mathrm{dL}$; aspartate aminotransferase (AST) $55 \mathrm{U} / \mathrm{L}$; alanine aminotransferase (ALT) $42 \mathrm{U} / \mathrm{L}$; alkaline phosphatase (ALP)
$500 \mathrm{U} / \mathrm{L}$; gamma-glutamyl transpeptidase (GGT) $264 \mathrm{U} / \mathrm{L}$; cholinesterase $5238 \mathrm{U} / \mathrm{L}$; total bilirubin $0,64 \mathrm{mg} / \mathrm{dL}$; alphafetoprotein (-FP) $319 \mathrm{ng} / \mathrm{mL}$. Gastroscopy showed esophageal varices grade F1-F2. Her Child-Pugh score was A, and Meld score was 7. Both contrast-enhanced ultrasonography (CEUS) and CT scan revealed an $8 \times 5 \times 7 \mathrm{~cm}$ sized arterial enhancing and delayed washout mass extended to the VII and VIII hepatic segments associated with underlying cirrhosis and without portal invasion, ascites, and splenomegaly. Tumor stage was Barcelona Clinic Liver Cancer (1) stage C.

According to the practice guidelines of the American Association for the Study of Liver Disease (AASLD), transarterial chemoembolization (TACE) with epirubicin-lipiodol was performed. Thirty days after TACE, a noncontrastenhanced CT showed no tumor shrinkage and a slight accumulation of lipiodol (pattern type IV). These data [4] evidenced an unsatisfactory response to TACE procedure. A hypofractionated stereotactic radiotherapy (HSRT) was performed. Patient was immobilized using the Elekta Stereotactic Body Frame which uses a rigid frame and a vacuum pillow and an abdominal compression device to reduce respiratory target motion. Treatment planning contrast-enhanced CT ( $3 \mathrm{~mm}$ slice thickness) was obtained to identify target volume visualization. According to our internal protocol based on the recent literature data (5), HSRT was delivered in 5 fractions for a total of 40 Gy on alternate days, and dose was prescribed to the $97 \%$ isodose line (Figure 1). As constrains for normal tissues, $33 \%$ and $50 \%$ of the whole liver volume received a total dose less than $21 \mathrm{~Gy}$ and $15 \mathrm{~Gy}$, respectively; 


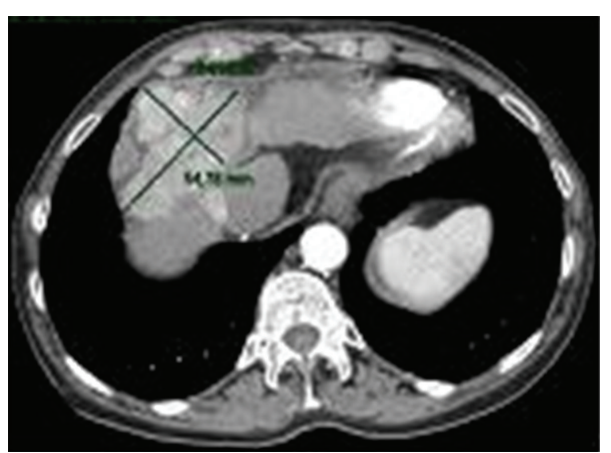

(a)

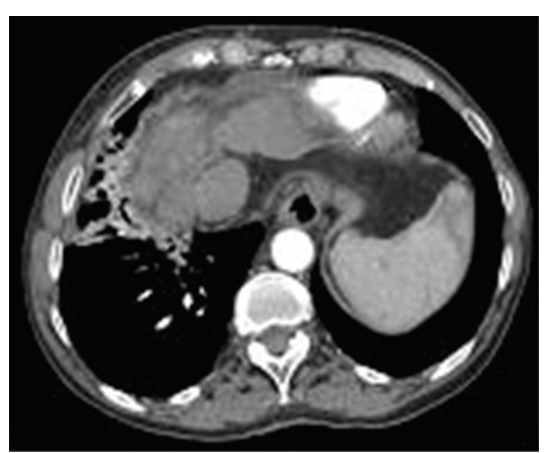

(b)

FIGURE 3: Abdominal computed tomography scans before and 6 months after HSRT show a complete disappearance of hypervascular arterial enhancement of primary tumour.

the percentage of each kidney volume to receive a total dose of $15 \mathrm{~Gy}$ was less than $35 \%$, while the maximum total dose did not exceed $27 \mathrm{~Gy}$ for the spinal cord and stomach/small intestine and $30 \mathrm{~Gy}$ for heart.

Planning was conducted on the Pinnacle 3D $8.0 \mathrm{~m}$ Version Planning System (Philips Healthcare, Best, Netherlands). The patient had no discomfort after the procedure, and no acute toxicity was reported.

At 1, 2, and 3 months after HSRT, ALP, GGT, and aFP decreased, respectively, to $411 \rightarrow 400 \rightarrow 361 \mathrm{U} / \mathrm{L}, 199 \rightarrow$ $156 \rightarrow 118 \mathrm{U} / \mathrm{L}$, and $107,6 \rightarrow 38 \rightarrow 19,8 \mathrm{ng} / \mathrm{mL}$; a further reduction of a-FP value at 7,4 ng/mL was observed within 6 months (Figure 2). CEUS at 1 and 2 months showed 3 large necrotic areas inside an unchanged size tumour mass. CT scan showed a $90 \%$ objective tumour response and a complete remission at 3 and 6 months after treatment, respectively (Figure 3).

Twenty-four months following HSRT, patient showed a multifocal intrahepatic dissemination without evidence of "in-field" local recurrence; therefore a systemic therapy with sorafenib was planned.

\section{Discussion}

According to AASLD, TACE is the first-line treatment in unresectable HCC. Nevertheless, it induces objective responses sustained for at least 6 months in only 35\% of patients [5]. Although TACE can be repeated in most patients, therapeutic efficacy cannot be expected by repetitive procedure, so that there is no consensus regarding the optimum treatment/retreatment strategy [6]. Conversely, repeated TACE treatments can result in deterioration of the liver function [7]. Failure of TACE treatment can be predicted with a CT scan 1 month after TACE, by volume measurement and by retention of lipiodol within tumor tissue [8]. For patients who have failed locoregional therapy, systemic therapy is the standard of care. However, another locoregional treatment can be performed in order to improve locoregional tumour control probability.

The role of external beam radiotherapy has always been limited by the low-dose tolerance of liver to radiation and subsequent high risk of RILD due to the high percentage of liver irradiated [9].
However, latest technological developments in RT planning and treatment delivery, such as HSRT, have allowed us to deliver higher radiation tumour dose while sparing surrounding normal tissues, suggesting a significant benefit for patients affected by intermediate or advanced HCC, alone or combined with TACE, in order to improve clinical results.

Despite the high size of gross tumour mass, our patient experienced a long-term complete remission (2 years) and a subsequent "out-field" intrahepatic dissemination that required systemic therapy. Probably, the peripheral tumour localization and the good liver reserve have enabled us to safely deliver a tumoricidal dose without occurring major side effects.

Using radiotherapy, several monoinstitutional experiences have shown promising results with a high rate of complete (50-60\%) and objective responses (75-80\%) as well as low rate of severe toxicity [10-13]. Furthermore, when stereotactic radiotherapy is used, the pattern of hepatic "outfield" recurrence compared to local or distant failure seems to be the main cause of progression disease influencing patient life expectancy.

\section{Conclusion}

Our experience with this case demonstrates that a complete remission of primary and a good cancer control can be obtained in local advanced HCC patients with HSRT. Two year following HSRT, a multifocal intrahepatic dissemination was diagnosed without evidence of the primary HCC. This confirms that the pattern of hepatic "out-field" recurrence is the main cause of HCC progression. HSRT, after TACE failure, was very effective in our patient, suggesting that HSRT might improve the overall survival rate and provides a good prognosis for patients with unresectable HCC.

\section{Consent}

Written informed consent was obtained from the patient for the publication of this case report and accompanying images. A copy of the written consent is available for review by the Editor-in-Chief of this journal. 


\section{Conflict of Interests}

The authors declare that they have no conflict of interests.

\section{Authors' Contribution}

F. Fiorica, S. Boccia, S. Sartori, A. Stefanelli, and S. Ursino were directly involved in the diagnostic workup and overall care of the patient. S. Ursino, F. Fiorica, and C. Greco drafted the manuscript. F. Cartei served as the lead clinician, selected the images presented, and obtained written consent from the patient. All authors read and approved the final paper.

\section{References}

[1] J. M. Llovet, C. Brú, and J. Bruix, "Prognosis of hepatocellular carcinoma: the BCLC staging classification," Seminars in Liver Disease, vol. 19, no. 3, pp. 329-337, 1999.

[2] C. Cammà, F. Schepis, A. Orlando et al., "Transarterial chemoembolization for unresectable hepatocellular carcinoma: meta-analysis of randomized controlled trials," Radiology, vol. 224, no. 1, pp. 47-54, 2002.

[3] S. Ursino, C. Greco, F. Cartei et al., "Radiotherapy and hepatocellular carcinoma: update and review of the literature," European Review for Medical and Pharmacological Sciences, vol. 16, no. 11, pp. 1599-1604, 2012.

[4] J. W. Zhang, X. Y. Feng, H. Q. Liu et al., "CT volume measurement for prognostic evaluation of unresectable hepatocellular carcinoma after TACE,' World Journal of Gastroenterology, vol. 16, no. 16, pp. 2038-2045, 2010.

[5] J. M. Llovet, M. I. Real, X. Montaña et al., "Arterial embolisation or chemoembolisation versus symptomatic treatment in patients with unresectable hepatocellular carcinoma: a randomised controlled trial," The Lancet, vol. 359, no. 9319, pp. 1734-1739, 2002.

[6] R. Lencioni, X. P. Chen, L. Dagher, and A. P. Venook, “Treatment of intermediate/advanced hepatocellular carcinoma in the clinic: how can outcomes be improved?” The Oncologist, vol. 15, supplement 4, pp. 42-52, 2010.

[7] K. Ahrar and S. Gupta, "Hepatic artery embolization for hepatocellular carcinoma: technique, patient selection, and outcomes," Surgical Oncology Clinics of North America, vol. 12, no. 1, pp. 105-126, 2003.

[8] K. Nishimine, H. Uchida, N. Matsuo et al., "Segmental transarterial chemoembolization with Lipiodol mixed with anticancer drugs for nonresectable hepatocellular carcinoma: follow-up CT and therapeutic results," Cancer Chemotherapy and Pharmacology, Supplement, vol. 33, no. 1, supplement, pp. S60-S68, 1994.

[9] R. A. Abrams, T. F. Pajak, T. L. Haulk, M. Flam, and S. O. Asbell, "Survival results among patients with $\alpha$-fetoproteinpositive, unresectable hepatocellular carcinoma: analysis of three sequential treatments of the RTOG and Johns Hopkins Oncology Center," Cancer Journal from Scientific American, vol. 4, no. 3, pp. 178-184, 1998.

[10] A. Takeda, M. Takahashi, E. Kunieda et al., "Hypofractionated stereotactic radiotherapy with and without transarterial chemoembolization for small hepatocellular carcinoma not eligible for other ablation therapies: preliminary results for efficacy and toxicity," Hepatology Research, vol. 38, no. 1, pp. 6069, 2008.
[11] J. H. Kwon, S. H. Bae, J. Y. Kim et al., "Long-term effect of stereotactic body radiation therapy for primary hepatocellular carcinoma ineligible for local ablation therapy or surgical resection. Stereotactic radiotherapy for liver cancer," BMC Cancer, vol. 10, article 475, 2010.

[12] J. K. Kang, M. S. Kim, C. K. Cho et al., "Stereotactic body radiation therapy for inoperable hepatocellular carcinoma as a local salvage treatment after incomplete transarterial chemoembolization," Cancer, vol. 118, no. 21, pp. 5424-5431, 2012.

[13] Y. Honda, T. Kimura, H. Aikata et al., "Stereotactic body radiation therapy combined with transcatheter arterial chemoembolization for small hepatocellular carcinoma," Journal of Gastroenterology and Hepatology, 2012. 


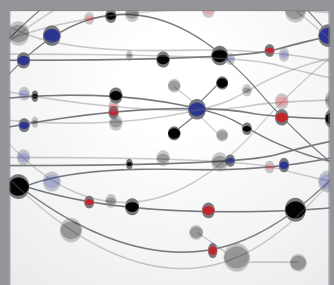

The Scientific World Journal
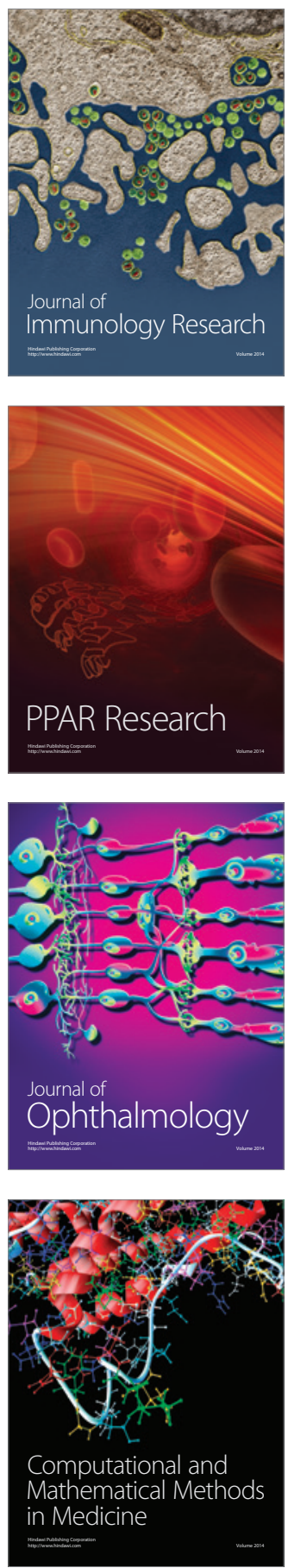

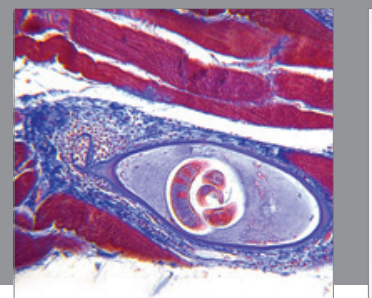

Gastroenterology

Research and Practice
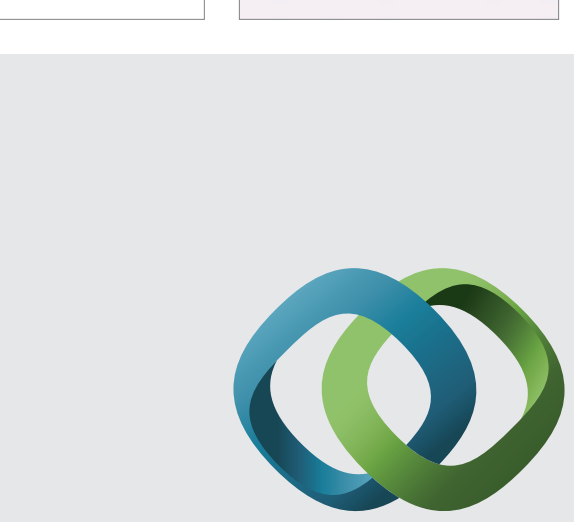

\section{Hindawi}

Submit your manuscripts at

http://www.hindawi.com
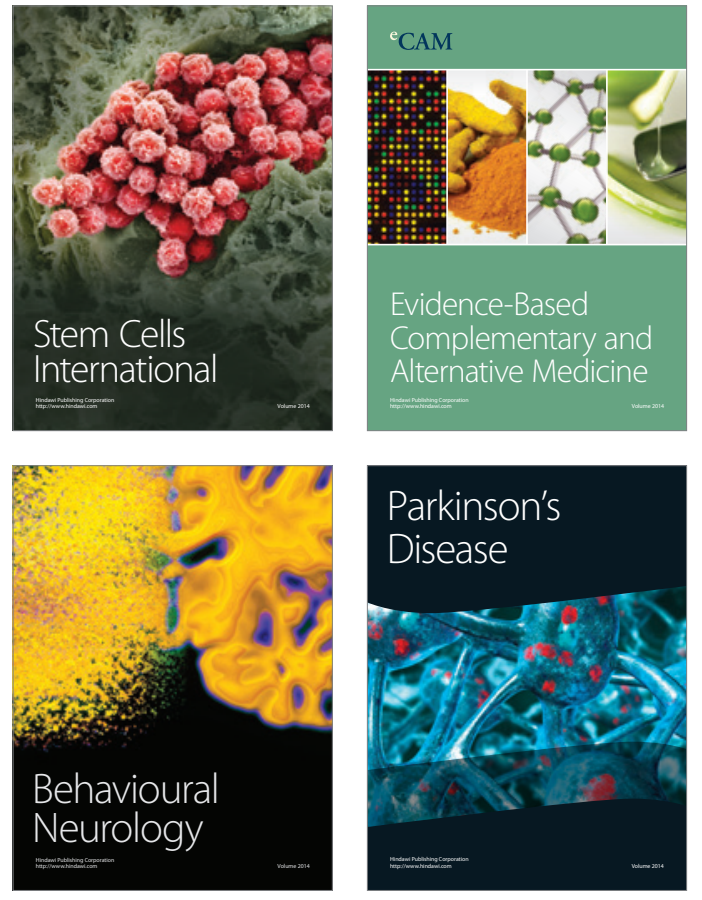
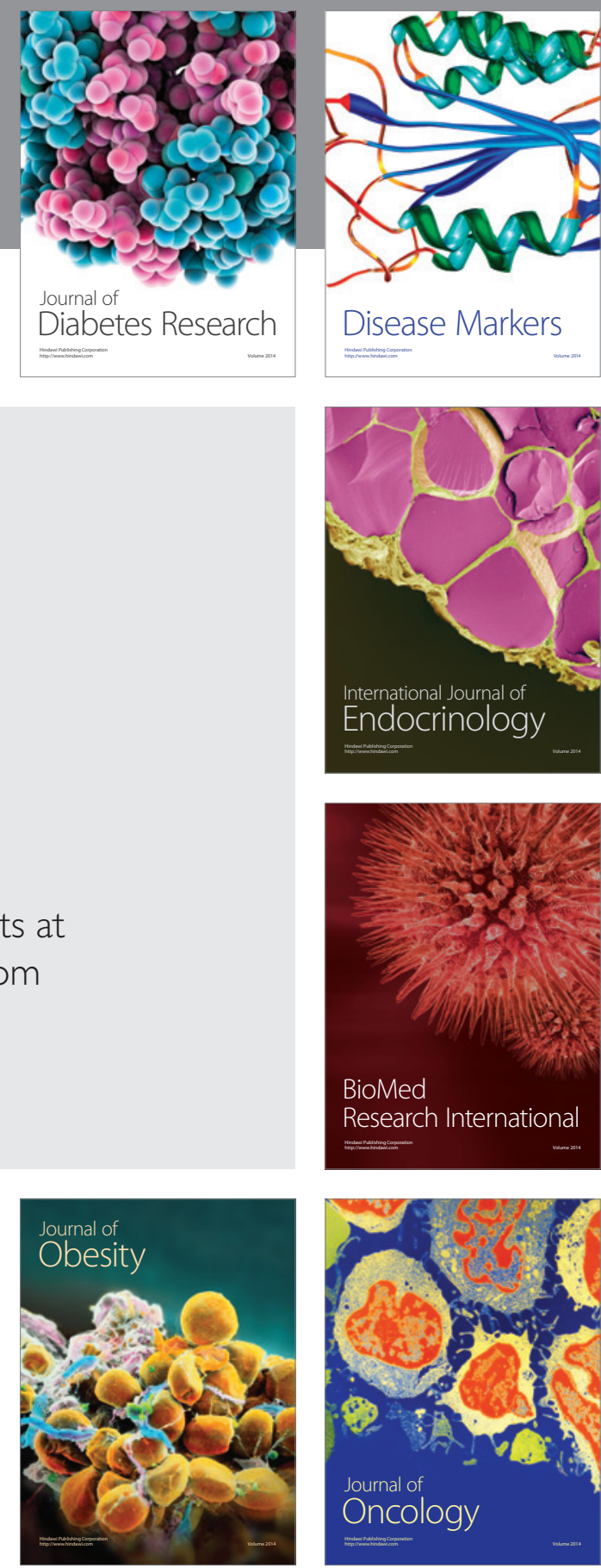

Disease Markers
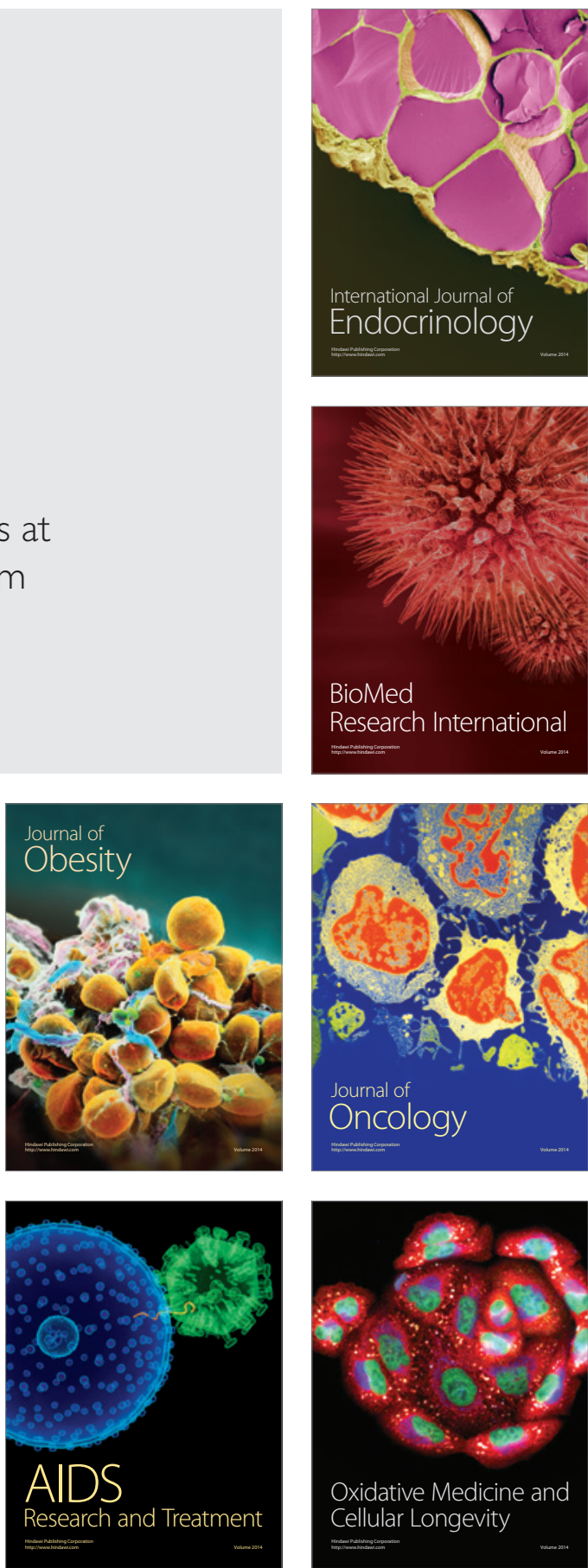